\title{
Sialylated galacto-oligosaccharides and 2'-fucosyllactose reduce necrotising enterocolitis in neonatal rats
}

\author{
Chloe A. Autran ${ }^{1}$, Margriet H. C. Schoterman ${ }^{2}$, Evelyn Jantscher-Krenn ${ }^{1}$, Johannis P. Kamerling ${ }^{3}$ \\ and Lars Bode ${ }^{1 *}$ \\ ${ }^{1}$ Division of Neonatology and Division of Gastroenterology, Hepatology and Nutrition, Department of Pediatrics, University of \\ California, San Diego, La Jolla, CA 92093, USA \\ ${ }^{2}$ FrieslandCampina, 3818 LE Amersfoort, The Netherlands \\ ${ }^{3}$ Microbial Physiology, Groningen Biomolecular Sciences and Biotechnology Institute, University of Groningen, 9747 AG \\ Groningen, The Netherlands
}

(Submitted 9 February 2016 - Final revision received 24 March 2016 - Accepted 11 April 2016)

\section{Abstract}

Necrotising enterocolitis (NEC) is one of the most frequent and fatal intestinal disorders in preterm infants and has very limited treatment options. Breast-fed infants are at a 6-10-fold lower NEC risk than formula-fed infants, and we have previously shown that human milk oligosaccharides (HMO) improved survival and reduced pathology in a rat NEC model. The HMO disialyllacto- $N$-tetraose (DSLNT) was most effective, and sialylation was shown to be essential for its protective effect. Galacto-oligosaccharides (GOS), currently added to some infant formula, but not containing sialic acid, had no effect. In addition to DSLNT, our previous work also showed that the neutral HMO fraction, which contains high concentrations of $2^{\prime}$-fucosyllactose ( $\left.2^{\prime} \mathrm{FL}\right)$, slightly improved pathology scores. Here, we assessed the in vivo efficacy of $2^{\prime} \mathrm{FL}$, as well as of GOS that we enzymatically sialylated (Sia-GOS). Neonatal rats were randomised into the following study groups - dam-fed (DF), formula-fed (FF), FF containing pooled HMO (10 mg/ml), GOS $(8 \mathrm{mg} / \mathrm{ml})$, Sia-GOS $(500 \mu \mathrm{m})$ or $2^{\prime} \mathrm{FL}(2 \mathrm{mg} / \mathrm{ml})-$ and subjected to the established NEC protocol. The DF and HMO groups had the lowest pathology scores with mean values of 0.67 (SD 0.34) and 0.90 (sD 0.47), respectively. The FF group had significantly elevated pathology scores of 2.02 (sD 0.63). Although the addition of GOS to the formula had no protective effect and generated scores of 2.00 (sD 0.63), the addition of Sia-GOS or 2'FL significantly lowered pathology scores to 1.32 (sD 0.56 ) $(P<0.0034)$ and 1.43 (sD 0.51) $(P<0.0040)$, respectively. The results warrant further studies to investigate the underlying mechanisms and to assess safety and efficacy in human neonates.

Key words: Human milk oligosaccharides: Galacto-oligosaccharides: 2'-Fucosyllactose: Necrotising enterocolitis: Premature infants: Infant formula

Necrotising enterocolitis (NEC) is one of the most common and often fatal intestinal disorders that affects nearly $10 \%$ of all very-low-birth weight (VLBW) infants ${ }^{(1)}$. About a quarter of the affected infants die from NEC, and the survivors are often faced with long-term neurological complications ${ }^{(2)}$. Breast-fed infants are at a 6-10-fold lower risk of developing NEC than formulafed $(\mathrm{FF})$ infants $^{(3,4)}$. However, the reasons for the benefits of breast milk remain elusive.

Human milk is an abundant source of oligosaccharides that are currently not present in infant formula ${ }^{(5)}$. We have recently shown that these human milk oligosaccharides (HMO) improve survival and reduce pathology scores in a preclinical NEC model in neonatal rats ${ }^{(6)}$. HMO are a group of more than 150 structurally distinct complex sugars, and we were able to identify one specific oligosaccharide named disialyllacto$N$-tetraose (DSLNT) that is most effective in preventing NEC in the rat model ${ }^{(6)}$. However, DSLNT is unique to human milk and, similar to most other HMO, currently not commercially available to supplement infant formula. Today, several infant formulas contain prebiotic oligosaccharides such as galactooligosaccharides (GOS) to mimic some of the beneficial effects of $\mathrm{HMO}^{(7)}$. In infants, consumption of formula enriched with GOS showed a significant increase in bifidobacteria and lactobacilli in the infant gut microbiota, which resembles that of breast-fed infants ${ }^{(8-10)}$. However, GOS are structurally different from HMO and do not contain sialic acid, which is an essential component for DSLNT to be effective in preventing NEC. Removal of just one sialic acid from DSLNT abrogates its

Abbreviations: 2'FL, 2'-fucosyllactose; DF, dam-fed; DP, degree of polymerisation; DSLNT, disialyllacto- $N$-tetraose; FF, formula-fed; GOS, galactooligosaccharides; HMO, human milk oligosaccharides; NEC, necrotising enterocolitis; Sia-GOS, sialylated galacto-oligosaccharides.

* Corresponding author: L. Bode, email lbode@ucsd.edu 
beneficial effects ${ }^{(6)}$. Although we have shown previously that GOS is ineffective in preventing disease in the neonatal rat model of $\mathrm{NEC}^{(6)}$, we now use the same model to assess whether or not modified GOS that is decorated with sialic acid ( $N$-acetylneuraminic acid (Neu5Ac) $)^{(11)}$ reduces NEC pathology.

In previous studies, the neutral HMO fraction, which contains high amounts of the HMO 2'-fucosyllactose ( $\left.2^{\prime} \mathrm{FL}\right)$, also showed a significant reduction in pathology scores in the rat NEC model $(P<0.05)^{(6)}$. Although not significant, $2^{\prime} \mathrm{FL}$ tended to decrease the incidence and severity of NEC in a preterm piglet model ${ }^{(12)}$. Therefore, we tested whether or not $2^{\prime} \mathrm{FL}$ reduces NEC pathology in the neonatal rat model.

\section{Methods}

\section{Source of galacto-oligosaccharides}

Vivinal $^{\circledR}$ GOS (FrieslandCampina), a mixture of GOS, is synthesised by the enzymatic transgalactosylation of cows' milkderived lactose using a $\beta$-galactosidase enzyme from Bacillus circulans. The syrup contains approximately 59\% GOS, $21 \%$ lactose, $19 \%$ glucose and $1 \%$ galactose on DM (75\%); in terms of the degree of polymerisation (DP), it contains approximately $20 \%$ DP1, $42 \%$ DP2, 24\% DP3, $10 \%$ DP 4 ; 3\% DP5 and $<0.5 \%$ DP6-DP9 ${ }^{(13)}$. Recently, the structures of $>40$ components up to DP5 have been reported ${ }^{(13)}$. A purified GOS material (97\% GOS, $0.7 \%$ galactose, $1.6 \%$ glucose and $0.7 \%$ lactose) was prepared from Vivinal ${ }^{\circledR}$ GOS by means of an enzymatic treatment with lactase to hydrolyse the lactose into glucose and galactose, after which the monosaccharides were removed by nanofiltration.

\section{Source and generation of sialylated galacto- oligosaccharides}

Sia-GOS (provided by Microbial Physiology, Groningen Biomolecular Sciences and Biotechnology Institute, University of Groningen) was synthesised by $\operatorname{Sia}(\alpha 2-3)$ transfer from donor $\kappa$-casein-derived glycomacropeptide (GMP; Sia $\geq 99 \%$ Neu 5 Ac) (provided by FrieslandCampina Innovation Centre) to acceptor Vivinal ${ }^{\circledR}$ GOS, using trans-sialidase from Trypanosoma cruzi as biocatalyst. The enzyme transfers $\operatorname{Sia}(\alpha 2-3)$ from a Sia $(\alpha 2-3) \mathrm{Gal}$ $(\beta 1-x)$ sequence on a donor to a $\beta$-linked galactose on an acceptor $^{(11)}$.

A solution of $5 \mathrm{~mm}(\alpha 2-3)$-linked Neu5Ac (2.5 g GMP), 2 mм Vivinal $^{\circledR}$ GOS (average DP of $3 ; 10 \% \mathrm{NaCl}$ was partially removed via a $3 \mathrm{kDa}$ cut-off filter) and $3.3 \mathrm{mU} / \mathrm{ml}$ recombinant T. cruzi trans-sialidase (TcTS) in $500 \mathrm{ml}$ of $50 \mathrm{~mm}$-sodium citrate $\mathrm{pH} 5$ was incubated for $48 \mathrm{~h}$ at $25^{\circ} \mathrm{C}^{(11)}$. Then, the reaction mixture was heated for $20 \mathrm{~min}$ at $60^{\circ} \mathrm{C}$, it was centrifuged for $15 \mathrm{~min}$ at $4500 \mathrm{~g}$ and filtered over a 3-kDa cut-off filter using a DiaFlow system (removal of (partially desialylated) GMP and TcTS). The obtained filtrate was lyophilised and separated (Sia-GOS, GOS, salt) on a Bio-Gel P-2 column $(60 \times 5 \mathrm{~cm})$ using Milli-Q water as eluent and a refractive-index detection system. The Sia-GOS-containing fractions, as traced by high-pH anionexchange chromatography with pulsed amperometric detection (HPAEC-PAD; Dionex ICS-3000 workstation) on a CarboPac
PA-1 column $(250 \times 4 \mathrm{~mm}$; gradient of 30-600 mm-sodium acetate in $0 \cdot 1 \mathrm{~m}-\mathrm{NaOH})^{(14)}$, were pooled and lyophilised. A further purification was carried out on a Dowex $1 \times 8$ column (formate form, $50 \times 1.5 \mathrm{~cm}$ ), eluted with Milli-Q water, followed by $50 \mathrm{~mm}$-ammonium bicarbonate. The Sia-GOS-containing fractions, eluted with ammonium bicarbonate, were pooled and lyophilised. The purity of Sia-GOS (as a colourless glass) was calculated to be $>95 \%$. Charge analysis on Resource Q with UV detection revealed mono-Sia-GOS-di-Sia-GOS in a molar ratio of $97: 3^{(11)}$. Fig. 1(a) presents the HPAEC-PAD profile of Sia-GOS and Fig. 1(b) shows its ${ }^{1} \mathrm{H}$ NMR spectrum.

\section{Source of 2'-fucosyllactose}

$2^{\prime}$ FL was produced by fermentation technology, and it was provided by FrieslandCampina Innovation Centre. The $2^{\prime} \mathrm{FL}$ product contained approximately $93 \% 2^{\prime} \mathrm{FL}, 3 \%$ glucose, $3 \%$ lactose and $1 \%$ sucrose.

\section{Source of human milk oligosaccharides}

Human milk was obtained from thirty-seven healthy volunteers of preterm infants recruited at the University of California, San Diego Medical Center, San Diego, CA, USA, after approval by the university's institutional review board. After centrifugation, the lipid layer was removed and proteins were precipitated from the aqueous phase by addition of two volumes of ice-cold
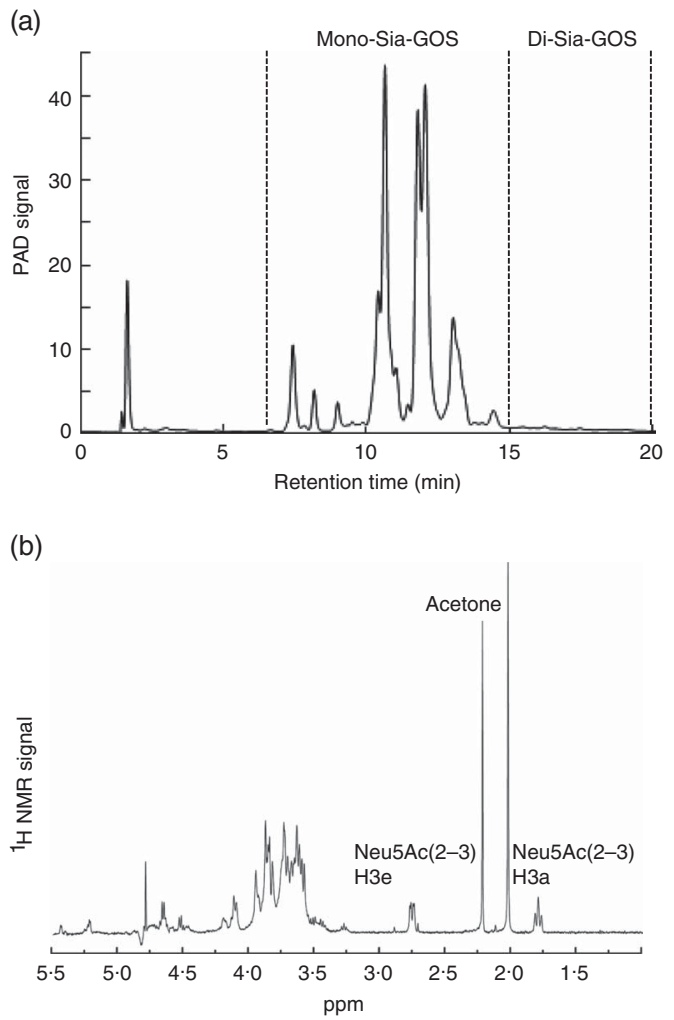

Fig. 1. Characterisation of sialylated galacto-oligosaccharides (Sia-GOS). (a) High-pH anion-exchange chromatography with pulsed amperometric detection (HPAEC-PAD) profile on CarboPac PA-1. Mono-Sia-GOS elutes between 7 and $15 \mathrm{~min}$ and di-Sia-GOS elutes between 15 and $20 \mathrm{~min}^{(11)}$. (b) ${ }^{1} \mathrm{H}$ NMR spectrum of Sia-GOS. Neu5Ac, $N$-acetyl-neuraminic acid; ppm, parts per million. 
Table 1. Composition of pooled human milk oligosaccharides (HMO)

\begin{tabular}{lc}
\hline HMO & Weight (\%) \\
\hline $2^{\prime}$-Fucosyllactose & $19 \cdot 8$ \\
3-Fucosyllactose & $4 \cdot 6$ \\
3'-Sialyllactose & $3 \cdot 9$ \\
$6^{\prime}$-Sialyllactose & $2 \cdot 1$ \\
Lacto- $N$-tetraose & 21.4 \\
Lacto- $N$-neotetraose & $4 \cdot 0$ \\
Lacto- $N$-fucopentaose I & $24 \cdot 3$ \\
Lacto- $N$-fucopentaose II & $5 \cdot 6$ \\
Lacto- $N$-fucopentaose III & 1.7 \\
Sialyllacto- $N$-tetraose b & $2 \cdot 2$ \\
Sialyllacto- $N$-tetraose c & 1.4 \\
Disialyllacto- $N$-tetraose & 4.7 \\
Fucosyllacto- $N$-hexaose & $<1$ \\
Difucosyllacto- $N$-hexaose & $<1$ \\
Fucosyl-disialyl-lacto- $N$-hexaose & $<1$ \\
Disialyl-lacto- $N$-hexaose & $<1$ \\
Uncharacterised HMO & 1.6 \\
Lactose & $<1$ \\
\hline
\end{tabular}

ethanol (100\%) and subsequent centrifugation. Ethanol was removed from the HMO-containing supernatant by rotoevaporation. Lactose and salts were removed by gel filtration chromatography over a Bio-Gel P-2 column $(100 \mathrm{~cm}$ length, $16 \mathrm{~mm}$ ID; Bio-Rad) using a semi-automated fast protein liquid chromatography system. The HMO composition (Table 1) was determined by HPLC and fluorescence detection after 2-aminobenzamide labelling. Peak annotation was based on standard retention times and MS analysis on a Thermo LCQ Duo Ion trap MS equipped with a Nano-ESI-source.

\section{Efficacy study in neonatal rat necrotising enterocolitis model}

As there are no in vitro models on NEC available, and human intervention studies are not feasible because of limited availability of HMO and other newly developed oligosaccharides, we used an NEC model in neonatal rats that was originally described by Barlow et al. and later modified and used to test HMO efficacy in the previous study ${ }^{(6)}$. In brief, pregnant timedated Sprague-Dawley rats were induced at term using Pitocin (1-2 U/animal). Immediately after birth, neonatal rats were randomised into one of the following six study groups: dam-fed as healthy control (DF; $n$ 18), formula-fed as disease control (FF; $n$ 22), FF containing pooled $\mathrm{HMO}$ at $10 \mathrm{mg} / \mathrm{ml}$ as positive intervention control (HMO; $n$ 15), FF containing GOS at $8 \mathrm{mg} /$ $\mathrm{ml}$ as negative intervention control (GOS; $n$ 15), FF containing Sia-GOS at $500 \mu \mathrm{M}$ (approximately $0.4 \mathrm{mg} / \mathrm{ml}$ ) as investigative intervention (Sia-GOS; $n$ 11) and FF containing $2^{\prime} \mathrm{FL}$ at $2 \mathrm{mg} / \mathrm{ml}$ as a second investigative intervention ( $\left.2^{\prime} \mathrm{FL} ; n 14\right)$. The dosage of HMO and $2^{\prime} \mathrm{FL}$ was based on the average content of these compounds in mature human milk. The dosage of Sia-GOS was based on the amount of DSLNT in human milk at 2-3 weeks postpartum, and on the dosage that was previously shown to be effective in improving survival and reducing pathology scores in the rat NEC model. The $8 \mathrm{mg}$ GOS/ml was based on the maximum amount of GOS, which is currently being used in infant formula.
Table 2. Composition of the study formula compared with rat milk

\begin{tabular}{lcr}
\hline & Study formula & Rat milk \\
\hline Protein $(\mathrm{g} / 100 \mathrm{ml})$ & 6.4 & 7.0 \\
Fat $(\mathrm{g} / 100 \mathrm{ml})$ & 12.2 & 14.6 \\
Lactose $(\mathrm{g} / 100 \mathrm{ml})$ & 7.9 & 2.8 \\
Energy $(\mathrm{kcal} / 100 \mathrm{ml})$ & 168.8 & 170.6 \\
\hline
\end{tabular}

* On day of life 5 and depending on the diet fed to the dam ${ }^{(15)}$.

Animals in the DF group remained with the dam. All other animals were separated from the dam, housed in a temperatureand humidity-controlled incubator and orally gavaged with a special rodent formula $(0.2 \mathrm{ml}$; without and with different oligosaccharides) twice daily. The formula approximates the protein and caloric content of rat breast milk (Table 2) and consists of $15 \mathrm{~g}$ of Similac 60/40 (Ross Pediatrics) in $75 \mathrm{ml}$ of Esbilac canine milk replacer (Pet-Ag). All animals, DF and gavaged, were exposed to $10 \mathrm{~min}$ of hypoxia $\left(5 \% \mathrm{O}_{2}, 95 \% \mathrm{~N}_{2}\right)$ three times daily in a modular chamber. All animals were killed $96 \mathrm{~h}$ postpartum; their intestines were collected and inspected for the presence of gross necrotic changes or pneumatosis intestinalis. A $0 \cdot 5-\mathrm{cm}$ section of the terminal ileum was prepared for H\&E staining per standard protocols and scored blindly by two investigators based on morphological changes that included epithelial sloughing, villus oedema, infiltration of neutrophils, apoptosis of villus enterocytes, crypt hyperplasia and misaligned nuclei in the epithelium. If at least one pathology sign was observed, a score of $0.5-1.5$ was assigned depending on severity. Two or three signs together resulted in a score of 2-3. The maximum score of 4 was given in case of complete obliteration of the epithelium with or without intestinal perforation. Studies were performed in two independent feeding experiments. The animal protocol was approved by The University of California San Diego Institutional Animal Care and Use Committee (IACUC), and complied with the Guide for the Care and Use of Laboratory Animals.

\section{Statistical analysis}

Pathology scores were plotted for each animal and the mean calculated per group. Each intervention was tested in at least two independent sets of experiments with a total of 11-22 animals per intervention group. Differences between all groups were calculated by one-way ANOVA with the Kruskal-Wallis test and Dunn's multiple comparison test. Differences between two groups were calculated with an unpaired, nonparametric Mann-Whitney test. Significance was defined as $P<0 \cdot 05$.

\section{Results}

Feeding formula without oligosaccharides increases necrotising enterocolitis pathology scores, but addition of human milk oligosaccharides prevents the increase in pathology scores

Consistent with previous studies ${ }^{(6)}$, most animals in the DF group showed no or very little sign of pathology with scores of $0,0.5$ or $1 \cdot 0$, and the group's mean pathology score was 0.67 (sD 0.34) (Fig. 2). Compared with the DF group, animals in the FF 
Oligosaccharides and necrotising enterocolitis

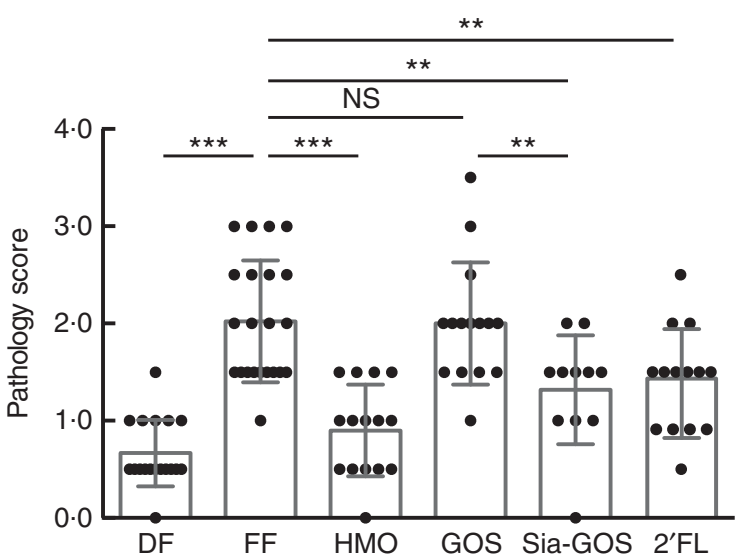

Fig. 2. Sialylated galacto-oligosaccharides (Sia-GOS) and 2'-fucosyllactose $\left(2^{\prime} \mathrm{FL}\right)$ reduce pathology scores in rat necrotising enterocolitis (NEC) model. Rat ileum pathology scores at $96 \mathrm{~h}$ postpartum (0: healthy; 4: complete destruction). Rat pups were either fed by the dam (DF) or separated from the dam and fed formula (FF) that was supplemented with human milk oligosaccharides (HMO, $10 \mathrm{mg} / \mathrm{ml})$, galacto-oligosaccharides $(\mathrm{GOS}, 8 \mathrm{mg} / \mathrm{ml})$, Sia-GOS $(500 \mu \mathrm{M}$ (approximately $0.4 \mathrm{mg} / \mathrm{ml}))$ or $2^{\prime} \mathrm{FL}(2 \mathrm{mg} / \mathrm{ml})$. Each intervention was tested in a total of eleven to twenty-two animals in two independent experiments. The pathology score for an individual animal. Values are mean pathology scores, and standard deviations are represented by bars and whiskers plots. ${ }^{\star *} P<0.01,{ }^{\star * \star} P<0.001$.

group that received formula without the addition of oligosaccharides had significantly higher pathology scores of 2.02 (SD 0.63) $(P<0.0001)$. Animals that received HMO with their formula had an average pathology score of 0.90 (SD 0.47$)$, which was significantly lower than the FF group $(P<0.0001)$ and not significantly different from the DF group $(P=0 \cdot 1267)$.

\section{Addition of unmodified galacto-oligosaccharides has no} effect on necrotising enterocolitis pathology scores, but sialylation of galacto-oligosaccharides reduces pathology scores significantly

As previously shown ${ }^{(6)}$, the addition of GOS to formula did not lower NEC pathology scores (2.00 (SD 0.63)) when compared with the FF group that received formula without the addition of oligosaccharides $(P=0.9895)$. However, when formula was supplemented with Sia-GOS, pathology scores were reduced to 1.32 (sD 0.56), which was significantly lower compared with the FF group $(P<0.01)$ and the GOS group $(P<0.01)$, but still significantly higher compared with the DF group $(P<0.001)$ and the HMO group $(P<0.05)$.

\section{Addition of 2'-fucosyllactose reduces necrotising enterocolitis pathology scores}

HMO are a heterogeneous group of oligosaccharides, and we have recently shown that an oligosaccharide that contained two sialic acids was most effective in reducing NEC pathology scores. Removal of just one sialic acid abrogated the protective effect $^{(6)}$. However, we also showed that a neutral HMO fraction that contains $>30 \% 2^{\prime} \mathrm{FL}$ also reduces NEC pathology scores. Thus, we added $2^{\prime} \mathrm{FL}$ alone as an intervention, leading to an average pathology score of 1.43 (SD 0.51), which was significantly lower than the scores in the FF group $(P<0.01)$ and the GOS group $(P<0.05)$, but again still significantly higher compared with the DF group $(P<0.0001)$ and the HMO group $(P<0.05)$, confirming that oligosaccharides other than $2^{\prime} \mathrm{FL}$ contribute to the protective effects of $\mathrm{HMO}$.

\section{Discussion}

Sia-GOS and 2'FL significantly reduced pathology scores in the neonatal rat model of NEC. However, the two interventions were less effective than the intervention with pooled $\mathrm{HMO}$, and pathology scores in the Sia-GOS and 2'FL groups were also still significantly higher than in the DF group.

As previously shown ${ }^{(6)}$, the FF group had elevated pathology scores, whereas the addition of unmodified GOS to the formula had no effect. Animals receiving Sia-GOS had significantly lower pathology scores than the FF and GOS-fed animals. This is in accordance with previous results showing that sialic acid is an essential component for the protective effect of DSLNT. Removal of both sialic acids, as well as removal of only the sialic acid from the terminal galactose from DSLNT, abrogated its protective effect $^{(6)}$. Sia-GOS as tested in this study is a mixture of monoand di-sialylated $\mathrm{GOS}^{(11)}$. As the potentially active disialylated GOS comprised only a very minor fraction of the Sia-GOS material (approximately 3\%), it may be active at very low concentrations. Future purification and structure elucidation is required to identify the active compound(s) within the Sia-GOS mixture and establish their dose-response relationships. As SiaGOS is not present in human milk, and infants are not exposed to it naturally, full safety and efficacy studies will be required.

$2^{\prime} \mathrm{FL}$, which also significantly reduced NEC pathology scores in the rat model, is in most cases a major constituent of human milk, and breast-fed infants are exposed to it naturally. However, there is currently no evidence to suggest that infants are more susceptible to develop NEC when they receive breast milk from non-secretor women who have an inactive fucosyltransferase 2 and do not secrete $2^{\prime} \mathrm{FL}$ with their milk. In a study in preterm piglets, addition of $5 \mathrm{~g} / 12^{\prime} \mathrm{FL}$ to infant formula tended to decrease incidence and severity of NEC, although bacterial colonisation and intestinal structural and functional parameters were not significantly affected ${ }^{(12)}$. Although these results from preclinical models are encouraging, neither the rat nor the piglet model fully represents NEC etiology and pathogenesis in human preterm infants. Clinical intervention studies will be required to assess whether or not $2^{\prime} \mathrm{FL}$ reduces NEC in preterm infants.

Until now, clinical intervention studies on the effects of other prebiotic oligosaccharides on NEC are also very limited and not yet unambiguous. An example is a clinical trial on the effects of a prebiotic (inulin), a probiotic (Bifidobacterium lactis) and the combination of the prebiotic and probiotic (synbiotic) added to human milk or formula on the prevention of NEC in VLBW infants $^{(16)}$. The probiotic and synbiotic decreased NEC, whereas the prebiotic inulin alone, which is not sialylated and structurally different from $2^{\prime} \mathrm{FL}$, did not show an effect. In another study, a prebiotic mixture of GOS and long chain fructooligosaccharides (ratio of 9:1) did reduce the incidence of NEC in VLBW infants who were exclusively breast-fed ${ }^{(17)}$. 
The underlying mechanisms for the beneficial effect of DSLNT, Sia-GOS and $2^{\prime} \mathrm{FL}$ on NEC remain unclear. Several potential mechanisms seem plausible. (1) Recent studies describe associations between gut microbiome and NEC onset ${ }^{(18,19)}$, and one can hypothesise that specific HMO such as DSLNT and 2'FL, as well as other oligosaccharides such as Sia-GOS, serve as structure-specific prebiotics and help shape a desirable gut microbiome or prevent NEC-associated dysbiosis. (2) HMO act as soluble decoy receptors that block the attachment of potential pathogens to the intestinal epithelial cell surface, preventing pathogen proliferation and invasion $^{(5)}$. However, there is currently no clear evidence that specific pathogens or combinations of pathogens are associated with $\operatorname{NEC}^{(19,20)}$. (3) Mucosal neutrophil infiltration is a hallmark during NEC pathogenesis, and HMO reduce selectin-mediated neutrophil infiltration and activation in vitro ${ }^{(21,22)}$. However, these effects are highly structure-specific and require that the effective oligosaccharides be both sialylated and fucosylated ${ }^{(23)}$. DSLNT and Sia-GOS are sialylated, but not fucosylated. 2'FL is fucosylated, but not sialylated. (4) HMO alter epithelial and immune cell responses ${ }^{(24-26)}$, which might affect NEC pathogenesis or improve tissue healing. These effects are highly structure-specific and likely receptor-mediated. We had previously shown that the effects of DSLNT are highly structure-specific, as the removal of just one sialic acid abrogates the protective effect ${ }^{(6)}$. We have also shown that specific synthetic DSLNT derivatives reduce NEC in the neonatal rat model of NEC while other derivatives show no effect $^{(27)}$, emphasising defined structural requirements. One can hypothesise that one or more active compounds in the Sia-GOS mixture fulfill these structural requirements and entertain the same receptor on epithelial or immune cells as DSLNT. However, $2^{\prime} \mathrm{FL}$ is structurally very different from DSLNT or Sia-GOS. $2^{\prime} \mathrm{FL}$ is not sialylated, but fucosylated. One can hypothesise that $2^{\prime} \mathrm{FL}$ acts through a mechanism different from DSLNT or Sia-GOS, either by using a different receptor on epithelial or immune cells or by using an entirely different route - for example serving as a structurespecific prebiotic.

As the administration of pooled HMO is consistently most effective in reducing pathology scores, providing human milk remains to be the best way to prevent premature infants from developing NEC. Available amounts of products such as SiaGOS and 2'FL are currently limited for use in infants, but new technologies to up-scale oligosaccharide production advance rapidly, which offers the possibility to use them in the future for preterm infants, who do not have access to human milk. Just recently, $2^{\prime}$ FL safety was established in preclinical and clinical studies $^{(28,29)}$, and has been registered as a novel ingredient for infant and toddler formula in Europe (Novel Food Application) and the USA (Generally Recognized As Safe registration). Further research is required to investigate the underlying mechanisms of how HMO and other oligosaccharides protect from NEC in preclinical models and to assess safety and efficacy of newly developed oligosaccharides in infants.

\section{Acknowledgements}

The authors thank M. H. Wilbrink, G. A. ten Kate and P. Sanders (Microbial Physiology, Groningen Biomolecular Sciences and Biotechnology Institute, University of Groningen) for making available Sia-GOS (project jointly financed by the European Union, European Regional Development Fund and the Dutch Ministry of Economic Affairs, Agriculture and Innovation, Peaks in the Delta, the Municipality of Groningen, and the Provinces of Groningen, Fryslan and Drenthe, as well as the Dutch Carbohydrate Competence Center).

Funding for this study was received from FrieslandCampina, Amersfoort, The Netherlands.

M. H. C. S., J. P. K. and L. B. designed the research; C. A. A., E. J.-K. and L. B. conducted the research; C. A. A., M. H. C. S. and L. B. analysed the data; M. H. C. S. and L. B. wrote the paper; M. H. C. S. and L. B. critically reviewed the paper; L. B. had primary responsibility for final content. All authors have read and approved the final manuscript.

M. H. C. S. is an employee of FrieslandCampina. FrieslandCampina donated the GOS and 2'FL used in this study. All other authors declare no conflicts of interest.

\section{References}

1. Neu J \& Walker WA (2011) Necrotizing enterocolitis. N Engl J Med 364, 255-264.

2. Rees CM, Pierro A \& Eaton S (2007) Neurodevelopmental outcomes of neonates with medically and surgically treated necrotizing enterocolitis. Arch Dis Child Fetal Neonatal Ed 92 , F193-F198.

3. Lucas A \& Cole TJ (1990) Breast milk and neonatal necrotising enterocolitis. Lancet 336, 1519-1523.

4. Meinzen-Derr J, Poindexter B, Wrage L, et al. (2009) Role of human milk in extremely low birth weight infants' risk of necrotizing enterocolitis or death. J Perinatol 29, 57-62.

5. Bode L (2012) Human milk oligosaccharides: every baby needs a sugar mama. Glycobiology 22, 1147-1162.

6. Jantscher-Krenn E, Zherebtsov M, Nissan C, et al. (2012) The human milk oligosaccharides disialyllactose-N-tetraose prevents necrotizing enterocolitis in neonatal rats. Gut $\mathbf{6 1}$, $1417-1425$.

7. Macfarlane GT, Steed H \& Macfarlane S (2008) Bacterial metabolism and health-related effects of galacto-oligosaccharides and other prebiotics. J Appl Microbiol 104, 305-344.

8. Fanaro S, Marten B, Bagna R, et al. (2009) Galactooligosaccharides are bifidogenic and safe at weaning: a double-blind randomized multicenter study. JPGN $\mathbf{4 8}$, $82-88$.

9. Ben XM, Zhou XY, Zhao WH, et al. (2004) Supplementation of milk formula with galacto-oligosaccharides improves intestinal micro-flora and fermentation in term infants. Chin Med J (Engl) 117, 927-931.

10. Vandenplas Y, Zakharova I \& Dmitrieva Y (2015) Oligosaccharides in infant formula: more evidence to validate the role of prebiotics. Br J Nutr 113, 1339-1344.

11. Wilbrink MH, ten Kate GA, Sanders P, et al. (2015) Enzymatic decoration of prebiotic galacto-oligosaccharides (Vivinal GOS) with sialic acid using Trypanosoma cruzi trans-sialidase and two bovine sialoglycoconjugates as donor substrates. I Agric Food Chem 63, 5976-5984.

12. Cilieborg MS, Jensen ML, Bering SB, et al. (2012) A milk oligosaccharide, 2'-fucosyllactose, may ameliorate necrotizing enterocolitis in preterm pigs. Breastfeeding Med 7, 571.

13. Van Leeuwen SS, Kuipers BJH, Dijkhuizen L, et al. (2014) ${ }^{1} \mathrm{H}$ NMR analysis of the lactose/ $\beta$-galactosidase-derived galactooligosaccharide components of Vivinal ${ }^{\circledR}$ GOS up to DP5. Corrigendum. Carbohydr Res 419, 69-70. 
14. Wilbrink MH, ten Kate GA, van Leeuwen SS, et al. (2014) Galactosyl-lactose sialylation using Trypanosoma cruzi transsialidase as the biocatalyst and bovine $\kappa$-casein-derived glycomacropeptide as the donor substrate. Appl Environ Microbiol 80, 5984-5991.

15. Nicholas KR \& Hartmann PE (1991) Milk secretion in the rat: progressive changes in milk composition during lactation and weaning and the effect of diet. Comp Biochem Physiol A Comp Physiol 98, 535-542.

16. Dilli D, Aydin B, Fettah ND, et al. (2015) The propre-save study: effects of probiotics and prebiotics alone or combined on necrotizting enterocolitis in very low birth weight infants. J Pediatr 166, 545-551.

17. Armanian AM, Sadeghnia A, Hoseinzadeh M, et al. (2014) The effect of neutral oligosaccharides on reducing the incidence of necrotizing enterocolitis in preterm infants: a randomized clinical trial. Int J Prev Med 5, 1387-1395.

18. Neu J (2013) The microbiome and its impact on disease in the preterm infant. Curr Pediatr Rep 1, 215-221.

19. Zhou Y, Shan G, Sodergren E, et al. (2015) Longitudinal analysis of the premature infant intestinal microbiome prior to necrotizing enterocolitis: a case-control study. PLOS ONE 10, e0118632.

20. Smith B, Bode S, Petersen BL, et al. (2011) Community analysis of bacteria colonizing intestinal tissue of neonates with necrotizing enterocolitis. BMC Microbiol 11, 1-12.

21. Bode L, Kunz C, Muhly-Reinholz M, et al. (2004) Inhibition of monocyte, lymphocyte, and neutrophil adhesion to endothelial cells by human milk oligosaccharides. Thromb Haemost 92, 1402-1410
22. Bode L, Rudloff S, Kunz C, et al. (2004) Human milk oligosaccharides reduce platelet-neutrophil complex formation leading to a decrease in neutrophil beta 2 integrin expression. J Leukoc Biol 76, 820-826.

23. Varki A (1997) Selectin ligands: will the real ones please stand up? J Clin Invest 99, 158-162.

24. Kuntz S, Kunz C \& Rudloff S (2009) Oligosaccharides from human milk induce growth arrest via G2/M by influencing growth-related cell cycle genes in intestinal epithelial cells. Br J Nutr 101, 1306-1315.

25. Comstock SS, Wang M, Hester SN, et al. (2013) Select human milk oligosaccharides directly modulate peripheral blood mononuclear cells isolated from 10-d-old pigs. Br J Nutr 111, $1-10$.

26. He Y, Liu S, Leone S, et al. (2014) Human colostrum oligosaccharides modulate major immunologic pathways of immature human intestine. Mucosal Immunol 7, 1326-1339.

27. Yu H, Lau K, Thon V, et al. (2014) Novel synthetic disialyl hexasaccharides protect neonatal rats from necrotizing enterocolitis. Angew Chem Int Ed Engl 53, 6687-6691.

28. Coulet M, Phothirath P, Allais L, et al. (2014) Pre-clinical safety evaluation of the synthetic human milk, nature-identical, oligosaccharide 2'-O-fucosyllactose (2'FL). Regul Toxicol Pharmacol 68, 59-69.

29. Marriage BJ, Buck RH, Goehring KC, et al. (2015) Infants fed a lower calorie formula with 2'FL show growth and 2'FL uptake like breast-fed infants. J Pediatr Gastroenterol Nutr $\mathbf{6 1}$, 649-658. 